\title{
El complejo de parasitoides de la polilla del brote del pino en Chile: pasado, presente y posible escenario futuro
}

\author{
The parasitoid complex of the pine shoot moth: past, present and probable future scenario \\ Mar Ramos Sanz ${ }^{a^{*}}$, Dolly Lanfranco Leverton ${ }^{b}$ \\ *Autor de correspondencia: a Universidad Austral de Chile, Facultad de Ciencias, Instituto de Zoología, \\ Valdivia, Chile, tel.: 63-221315, mar.ramos@ postgrado.uach.cl \\ bUniversidad Austral de Chile, Facultad de Ciencias Forestales y Recursos Naturales, Valdivia, Chile
}

\section{SUMMARY}

The classical biological control, or the introduction and release of different exotic biocontrol agents to control introduced insect pests, is one of the most currently used methods for decreasing insect pest populations. In Chile the use of these methods for "the European pine shoot moth", Rhyacionia buoliana (Lepidoptera: Tortricidae), was a pioneer decision that supposed the accomplishment of forest management and the implementation of research that permitted the generation of action plans. Today, twenty years after the introduction of Orgilus obscurator (Hymenoptera: Braconidae) as the main parasitoid for control of this pest, the current situation is more complex than expected, with the presence of other native parasitoids and hyperparasitoids. In this work the evolution of the pine shoot moth control in Chile since the detection of the pest in 1985, the beginning of the biological control exerted by $O$. obscurator, and the present and probable future of this control, will be analyzed.

Key words: classical biological control, European pine shoot moth, Orgilus obscurator.

\section{RESUMEN}

El control biológico clásico o la introducción y liberación de biocontroladores exóticos es una de las técnicas más utilizadas en la actualidad para reducir las poblaciones de insectos plaga introducidas. En Chile, el uso de esta técnica fue una decisión pionera para controlar la polilla del brote del pino, Rhyacionia buoliana (Lepidoptera: Tortricidae), que supuso la ejecución de medidas de manejo forestal y la generación de investigación que permitió implementar planes de acción. A veinte años de la introducción de Orgilus obscurator (Hymenoptera: Braconidae) como parasitoide principal de esta plaga, el escenario es más complejo de lo esperado, con la aparición de nuevos parasitoides nativos e hiperparasitoides introducidos. En este trabajo se analiza la evolución que el control biológico de $R$. buoliana ha tenido en Chile desde el momento de su detección en 1985, el comienzo del control biológico ejercido por $O$. obscurator, y el presente y posible futuro de dicho control.

Palabras clave: control biológico clásico, polilla del brote, Orgilus obscurator.

\section{INTRODUCCIÓN}

El concepto de control biológico asociado a insectos y plantas plaga se define, en sentido amplio, como la acción de parásitos, depredadores y patógenos que actúan disminuyendo la densidad poblacional de una especie plaga dada, pero nunca acabando con ella del todo; o más concretamente, en el caso de insectos plaga se considera el método más apropiado para reducir el número de especies dañinas por debajo de un umbral económico dado (McFadyen 1998, Day 2005, van Lenteren et al. 2006).

Esta amplia definición comprende tres técnicas o métodos diferentes: a) conservación o mantenimiento de las poblaciones de biocontroladores nativos, b) aumento de las poblaciones de biocontroladores exóticos o nativos a través de su monitoreo y manipulación (fundamentalmente cría en laboratorio y liberación inundativa o inoculativa en el medio) y c) control biológico clásico, conocido como la importación y liberación de diferentes organismos, generalmente controladores biológicos exóticos, cuya eficacia ante la misma plaga en otros países permitiría esperar en el país donde es introducido idénticos resultados, así como el establecimiento de sus propias poblaciones (Howarth 1991, McFadyen 1998, Tezze y Botto 2004, Day 2005, Kehrli et al. 2005, Mills 2005).

El aumento en la crítica hacia los sistemas de control químico de plagas, tales como los plaguicidas, que aumentan la resistencia de las plagas así como elevan los niveles de contaminación, provocó un entusiasmo desmedido por la introducción de controladores biológicos, ya que muchos de sus seguidores lo consideran un sistema de control eficaz y conservacionista con el medio ambiente (Howarth 1991, Simberloff y Stiling 1996, McFadyen 1998). 
A pesar de la eficacia confirmada del control biológico (Howarth 1991, Cruttwell 1998, Mills 2005, Stiling y Cornelissen 2005), actualmente ha sido muy cuestionada su validez en relación a los riesgos implicados en su uso, debido a que la mayoría de los programas de control biológico exitosos o no introducen en el ecosistema especies exóticas que pueden tener algún efecto directo o indirecto sobre la entomofauna, incluyendo la plaga y sus agentes de control en el futuro (Simberloff y Stiling 1996, Louda et al. 1997, Boettner et al. 2000, Hoffman et al. 2002, Linch et al. 2002, Hoelmer y Kirk 2005, Mills 2005, van Lenteren et al. 2006).

Uno de los mayores efectos del control biológico es la pérdida de poblaciones de enemigos naturales nativos que se adaptan a la plaga, en parte porque en general los parasitoides nativos no son específicos de la plaga introducida y actúan como generalistas, de manera que no son capaces de responder ante altas densidades poblacionales de las especies plaga y se ven desplazados por el controlador introducido. Éste es un tema emergente, puesto que altera la diversidad de los sistemas y complica los programas de facilitación del hábitat (Kehrli et al. 2005, van Lenteren et al. 2006).

La introducción de controladores biológicos es sólo parte de un problema mayor: la invasión de nuevas áreas por especies foráneas, reconocida a su vez como uno de los factores relevantes en los procesos de extinción de especies nativas (Howarth 1991, Parker et al. 2006).

El uso de modelos también ayuda a conocer el estado de las poblaciones de la plaga y de sus parasitoides, lo que con datos de monitoreos continuados (series de tiempo), es fundamental para evaluar el éxito o fracaso del control biológico y predecir sus posibles cambios (Hawkins et al. 1993, Louda et al. 1997, Mills 2005).

En este trabajo se revisa el complejo de parasitoides asociados a la polilla del brote del pino, Rhyacionia buoliana (Schiff) (Lepidoptera: Tortricidae), analizándose la estructura y evolución del complejo en Chile desde que se decidiera aplicar control biológico.

\section{ANTECEDENTES HISTÓRICOS DEL PROBLEMA}

Actualmente Chile posee cerca de dos millones de hectáreas de bosques artificiales comerciales constituidos por especies de rápido crecimiento. Entre ellos destacan los bosques de Pinus radiata D. Don, distribuidos desde la región de Valparaíso a la región de Los Lagos, con una extensión de aproximadamente 1,5 millones de hectáreas. En un principio, $P$. radiata fue plantado con fines ornamentales, pero a comienzos del siglo XX se utilizó para otros fines como en las minas de carbón de Lota entre 1930-1935. A partir de 1950 la expansión de plantaciones de $P$. radiata aumentó de manera exponencial, sobre todo en la década de los 70 como consecuencia del llamado "Plan de Reforestación", desarrollado por el Ministerio de
Agricultura. Este plan, junto con los subsidios establecidos a partir de 1974 por el Decreto Ley 701, incrementó de manera notable la superficie de plantaciones, principalmente de $P$. radiata desde la región del Maule a la región de Los Lagos (Donoso y Lara 1996, Gayoso 1996).

La extensión y composición de estos bosques artificiales situó a $P$. radiata como especie altamente vulnerable ante el ataque de plagas. Así, en 1985 se detectó en Chile a $R$. buoliana, la polilla del brote del pino, que en muy poco tiempo colonizó y se estableció en las plantaciones de la región de Los Ríos y desde ahí se expandió hacia el norte, encontrándose actualmente entre las regiones de Valparaíso y de Los Lagos, con variados grados de infestación. Estas fluctuaciones se deben a diversos factores como el clima, las condiciones de sitio, la calidad de los árboles, los cambios poblacionales de la plaga y la influencia de los agentes de control (Cerda et al. 1985, 1986, Ide y Lanfranco 1994).

El comportamiento de $R$. buoliana en Chile es monovoltino, aunque hacia las regiones del norte del país, donde el clima es más cálido, el insecto adelanta su emergencia como adulto y su periodo de vuelo respecto a las zonas más templadas del sur, sin llegar a comportarse como bivoltino (Aguilar 2001). La emergencia de los adultos se produce desde mediados de noviembre hasta la primera semana de febrero, viviendo éstos en promedio unos 12 días, aunque en latitudes más bajas la emergencia se produce a principios de octubre, debido probablemente a la mayor temperatura (Cerda et al. 1986, Huerta y Pérez 1997, Aguilar 2001). Los adultos se aparean durante las primeras 24 horas de vida y comienzan a oviponer. Los huevos son depositados en los brotes en distintos estratos del árbol. El principal daño efectuado por $R$. buoliana se produce durante los estadíos larvales. El primer estadío larval se introduce en la parte inferior de las yemas de $P$. radiata, donde construyen un pequeño capullo de seda, después penetran en la base, horadándola y provocando escurrimientos de resina, como mecanismo de defensa del árbol. En el segundo estadío, las larvas migran y perforan las yemas, construyendo su nuevo nicho con sedas y heces. En estas condiciones, las larvas de segundo y tercer estadío pasan el invierno, permaneciendo inactivas y sin alimentarse. El cuarto estadío larval migra en primavera a un brote más suculento, donde terminan su ciclo provocando el mayor daño. Finalmente, entre mediados de septiembre y principios de diciembre se transforman en pupa, construyendo su capullo en el interior del brote, el cual es recubierto por un grumo de resina (Robredo 1978, INIA/SAG 1993, Huerta y Pérez 1997, Aguilar 2001, Heeley et al. 2003).

Aunque $R$. buoliana no provoca la muerte del árbol, infestaciones sucesivas a nivel apical ocasionan deformaciones fustales y pérdidas de crecimiento en altura y diámetro, que afectan la calidad de la madera y su rendimiento. Las plantaciones con mayor susceptibilidad al ataque son las de uno a cuatro años (con alturas cercanas a los $8 \mathrm{~m}$ ), según los niveles poblacionales de $R$. buoliana, y no los árboles 
mayores de 15 años, tal como se señala en la bibliografía referente a la biología del insecto fuera de Chile (Robredo 1978, Lanfranco et al. 1991, 1994, INIA/SAG 1993, Ide y Lanfranco 1994).

La aparición de $R$. buoliana en Chile no sólo supuso pérdidas económicas para el país y más concretamente para el sector forestal, sino que esta especie llegó a ser la plaga más importante, suponiendo a su vez un hito, ya que en ningún otro país con presencia de la misma en Pinus spp. se observó el número de individuos por árbol y la magnitud del daño alcanzado en Chile (Lanfranco et al. 1991, Ide y Lanfranco 1994, 2001).

La primera respuesta de las autoridades fue el ataque directo hacia las poblaciones de $R$. buoliana mediante la aplicación de insecticidas (Cerda et al. 1988). Como este método de control no resultó efectivo, se planteó el control de la plaga utilizando control biológico clásico (Howarth 1991, van Driesche y Bellows 1996, McFadyen 1998, Day 2005).

Después de estudios paralelos realizados por organismos oficiales como el Instituto Nacional de Investigaciones Agropecuarias (INIA) y el Servicio Agrícola y Ganadero (SAG), y con asesoría de investigadores europeos con experiencia en control de plagas, y más concretamente en el control de $R$. buoliana, se introdujo el parasitoide Orgilus obscurator Ness (Hymenoptera: Braconidae) (Lanfranco et al. 1991, INIA/SAG 1993, Ide y Lanfranco 1994, 2001).

\section{COMPLEJO PARASITOIDE ASOCIADO}

Rhyacionia buoliana, al igual que otros lepidópteros, constituye un rico complejo alimenticio que atrae especies parasitoides que la atacan en distintos estados de su ciclo y difieren en su modo de desarrollo (Mills 2005). Por ello, además de $O$. obscurator, con el tiempo se detectaron otros parasitoides nativos e introducidos que ayudaban en el control de $R$. buoliana y que, a su vez, constituían un complejo de parasitoides y depredadores que actuaban en distinto tiempo y distintos estados de $R$. buoliana. A continuación se destacan algunos de los parasitoides más importantes, por estado de desarrollo (parasitoides de huevos, parasitoides de larvas de los estadíos L1 y L2, de los estadíos L3 y L4, de los estadíos L5, L6, de pupas, adultos y depredadores asociados).

Parasitoides de huevos. Se conocen cuatro especies de tricogrammátidos (Hymenoptera: Trichogrammatidae) que actúan parasitando los huevos de $R$. buoliana, de las cuales tres son nativas: Trichogramma exiguum Pinto y Platner, Trichogramma pretiosum Riley y Trichogramma nerudai Pintureau y Gerding; y una introducida, Trichogramma embryophagum Harting (Pintureau et al. 1999). La utilización de estas especies en el control de $R$. buoliana fundamentalmente ha sido llevada a cabo mediante liberaciones inundativas (BIOforest 1995, Gerding y Torres 2001, Tezze y Botto 2004, Torres y Gerding 2005). A pesar de los buenos resultados presentados en laboratorio bajo condiciones controladas, el efecto de las cuatro especies sobre las poblaciones naturales varía entre 50 y $60 \%$, principalmente debido a su limitada capacidad de búsqueda (las hembras de Trichogramma spp. atacan prácticamente cualquier huevo de lepidóptero existente). Además, los huevos de $R$. buoliana se encuentran aislados y existe una alta mortalidad de los individuos adultos de los tricogrammátidos, asociada probablemente al efecto de la temperatura y humedad (BIOforest 1995, Smith 1996, Gerding y Torres 2001, Tezze y Botto 2004). En ciertas zonas de la Patagonia argentina sólo T. nerudai ha generado buenos resultados (Tezze y Botto 2004). Actualmente se liberan adultos de T. nerudai a modo de biopesticida sobre plantaciones de $P$. radiata, Malus domestica Borkh y cultivos de Solanum lycopersicum L. y Zea mays L. para controlar las poblaciones de sus respectivas plagas ( $R$. buoliana, Cydia pomonella (Linnaeus) (Lepidoptera; Tortricidae), Tuta absoluta (Meyrick) (Lepidoptera: Gelechiidae) y Helicoverpa zea (Boddie) (Lepidoptera: Noctuidae)), además de otras especies plaga de lepidópteros. Los niveles de control sobre $R$. buoliana en laboratorio oscilan entre 30 y $90 \%$ (BIOforest 1995). Sin embargo, es fundamental monitorear permanentemente estas especies y reajustar el número de individuos liberados para obtener resultados duraderos (Arthur y Juillet 1961, BIOforest 1995, Botto 1998, Gerding y Torres 2001, Tezze y Botto 2004, Torres y Gerding 2005).

Parasitoides de los primeros estadíos (L1 y L2). Dos parasitoides primarios actúan sobre los primeros estadíos larvales, uno es Orgilus obscurator (Ness), del cual se liberan hembras apareadas o se implantan larvas parasitadas, lo que se realiza desde hace unos 10 años, y el segundo, un ichneumónido endoparasitoide koinobionte del género Temelucha (Foerster) (Hymenoptera: Ichneumonidae). Esta especie parasitoide es el principal competidor de $O$. obscurator y se trata de una especie nativa de Argentina, Temelucha platensis Brèthes, y nueva especie presente en Chile. Además se determinó que no se trata de Temelucha interruptor (Grav.), especie de origen europeo que está ligada al complejo parasitoide de $R$. buoliana (Brèthes 1917, Juillet 1960, Arthur y Juillet 1961, Arthur et al. 1964, Townes 1971, Schröder 1974, Huerta et al. 2004, Ide et al. 2007).

La especie principal de este complejo es Orgilus obscurator, un endoparasitoide solitario y koinobionte, del que cabe destacar su alta especificidad, fecundidad, eficacia en la búsqueda de hospederos y resistencia a temperaturas bajas (Arthur y Juillet 1961, Schröder 1974, INIA/SAG 1993, Gauld y Bolton 1988, Howarth 1991, Lanfranco et al. 1991, 1994, Gauld y Shaw 1995, van Driesche y Bellows 1996, McFadyen 1998, Ide y Lanfranco 2001). 
Tanto $O$. obscurator como T. platensis actúan en los primeros estadíos larvales (L1 y L2) de $R$. buoliana. Ambas especies son solitarias y sus hembras introducen con el ovipositor el huevo en el hemocele. Sus larvas son fácilmente distinguibles entre sí. Ambas poseen tres estadíos larvales, y un último estado de pupa que se desarrolla en el exterior del hospedero ya muerto. Las pupas también exhiben diferencias morfológicas y en los tiempos de desarrollo, pupando T. platensis 15-20 días antes que $O$. obscurator (Arthur y Juillet 1961, Schröder 1974, Gauld y Shaw 1995, Lanfranco et al. 1991, Huerta et al. 2004, Ide et al. 2007).

En esta interacción, T. platensis puede actuar como endoparasitoide koinobionte o como cleptoparasitoide y, por lo tanto, la búsqueda de las hembras está dirigida hacia hospederos previamente parasitados por $O$. obscurator o como competidor intragremial, atacando tanto a $R$. buoliana como a $O$. obscurator, siendo el tipo de búsqueda distinto y probablemente relacionado con diferencias en las densidades poblacionales de cada una de las especies (Taylor 1988, Hunter et al. 2002, Hougardy y Grégoire 2003, Jaloux et al. 2005, 2007, Jaloux y Monge 2006).

Existe una tercera especie en este complejo que actúa durante los primeros estadíos larvales de $R$. buoliana y que fue detectada en 2005. Perilampus tristis Mayr (Hymenoptera: Perilampidae), aumenta entonces la complejidad en las relaciones entre las dos especies de parasitoides primarios. Es un hiperparasitoide, al igual que gran parte de las especies pertenecientes al género Perilampus. Las hembras de este género ovipositan en material vegetal cercano a las larvas de su hospedero y de dichos huevos salen larvas planidiales que penetran en su interior, introduciéndose dentro del hemocele y atacando a los parasitoides primarios que se encuentran dentro, o bien esperan a que éstos penetren para atacarlos (Smith 1912, Parker 1924, Clausen 1940, Schröder 1974, Heraty y Darling 1984, Tanton y Epila 1984, Gauld y Bolton 1988, Hanson y LaSalle 1995, Darling 1996, Darling y Roberts 1999).

Actualmente $P$. tristis, de origen paleártico, tiene una distribución mundial y existe cierta controversia en torno a su rango de hospederos, ya que se la puede encontrar citada en relación a especies de parasitoides primarios de $C$. pomonella y asociada a parasitoides primarios de $R$. buoliana. En Chile esta especie ha sido recientemente asociada al complejo formado por $O$. obscurator y $T$. platensis en $R$. buoliana, aunque aún se desconoce si tiene la capacidad de asociarse a otros complejos de parasitoides (Schröder 1974, Darling 1996, Mills 2005).

El carácter hiperparasitoide de esta especie la sitúa en el tope de esta cadena trófica, aunque se desconoce su comportamiento actual, si es un hiperparasitoide obligatorio o facultativo, sus rasgos de historia de vida, comportamiento de búsqueda, entre otros. El análisis y estudio de dichos comportamientos podrían ayudar a conocer las interacciones básicas de los niveles que componen esta cadena trófica y la capacidad de $P$. tristis para influir en las poblaciones de parasitoides primarios ejerciendo un control de tipo top-down sobre las mismas (Schooler et al. 1996, Müller et al. 1999, Sullivan y Völkl 1999, Buitenhuis et al. 2005, van Veen et al. 2006).

Además de estas tres especies, se tienen registros esporádicos de poca incidencia de especies nativas como Anacis rubripes (Spinola) (Hymenoptera: Ichneumonidae) (Lanfranco et al. 1991, 1994).

Los roles desempeñados tanto por $P$. tristis como por T. platensis pueden ser clave en este sistema multiespecífico, ya que se desconoce el tipo de estrategia parasitoide de ambas especies, si son endoparasitoides, cleptoparasitoides o competidores intragremiales (en el caso de T. platensis), si actúan como depredadores tope o como competidores facultativos (en el caso de P. tristis) y, sobre todo, el efecto que tienen en las poblaciones del principal parasitoide primario $O$. obscurator y en la especie plaga $R$. buoliana (figura 1).

Parasitoides de los estadíos L3 y L4. Durante los estadíos 3 y 4 las larvas de $R$. buoliana permanecen inactivas, refugiadas en el interior de los brotes. Se desconocen parasitoides que actúen en esta fase. No obstante, los biocontroladores nativos conocidos ya han parasitado previamente estas larvas en estadíos anteriores. Otro tanto ocurre con los parasitoides introducidos.

Parasitoides de los estadíos L5, L6 y pupas. Son varias las especies conocidas actuando entre los últimos estadíos larvales y el estado de pupa, entre las que cabe destacar por orden de aparición: Neocryptopterix hypodyneri (Porter) (Hymenoptera: Ichneumonidae), Phorocera casanuevai Cortés (Diptera: Tachinidae), Incamya chilensis Aldrich (Diptera:

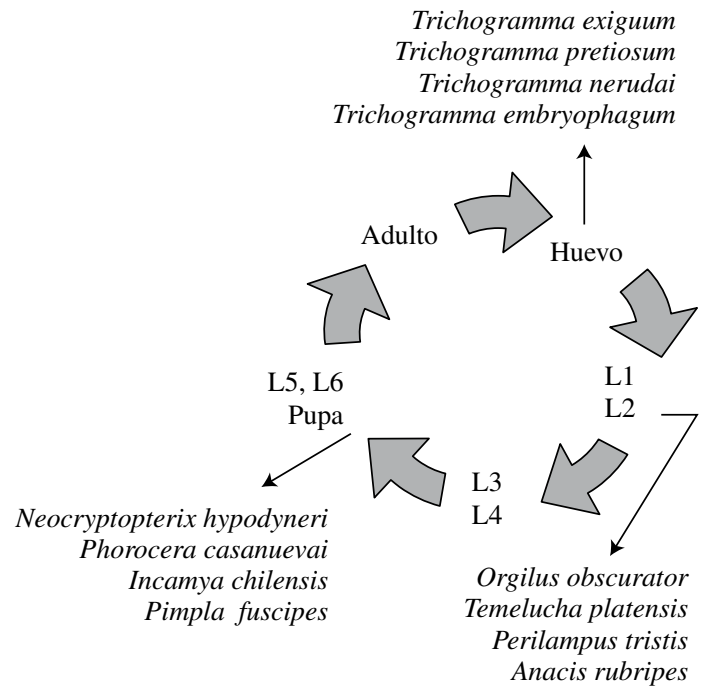

Figura 1. Parasitoides que actúan en el estado de huevo y en los estadíos larvales de Rhyacionia buoliana.

Parasitoids of eggs and larval instars of Rhyacionia buoliana. 
Tachinidae) y Pimpla fuscipes (Brullé) (Hymenoptera: Ichneumonidae) (Lanfranco et al. 1991, 1994).

La importancia en el control biológico de $R$. buoliana de estas especies es mínima y éstas actúan completando o ayudando en el control de las poblaciones. Neocryptopterix hypodyneri, I. chilensis y Ph. casanuevai son parasitoides koinobiontes que actúan entre el estadío larval 5 y 6 , aunque I. chilensis entra en el estado de pupa; son generalistas y por ello no suponen un riesgo para $O$. obscurator en términos competitivos (Lanfranco et al. 1991, 1994).

Cabe destacar la especie $P$. fuscipes, ya que aparece en un importante número de predios, no obstante, su parasitismo oscila entre 2 y $50 \%$. Pimpla fuscipes es un endoparasitoide idiobionte que ataca a prepupas y pupas de $R$. buoliana $\mathrm{y}$, por lo tanto, su desarrollo es muy rápido. Aunque en un principio se pueda pensar que actuaría compitiendo con el complejo anterior (Orgilus-Temelucha-Perilampus), $P$. fuscipes discrimina entre las pupas de R. buoliana no parasitadas y las pupas de Temelucha sp. y Orgilus sp. y es generalista, sirviendo como complemento más que como competidor (Lanfranco y Cerda 1987, Lanfranco et al. 1991, 1994, Wahl y Gauld 1998).

Parasitoides de adultos. No hay evidencia de parasitoides que actúen en este estado (Arthur y Juliet 1961, Huerta et al. 2004).

Depredadores asociados. Los depredadores asociados a este sistema merecen mención aparte, pues producen en mayor o menor medida una mortalidad entre el 5 y el $14 \%$, como es el caso del jilguero Carduelis barbata (Mol.) (Fringilidae), que consume larvas de los últimos estadíos, debido a que en esos momentos se observan fácilmente los grumos de resina producidos por la planta en respuesta al ataque de las larvas. La tasa máxima de consumo por $C$. barbata fue de 21-45 larvas por día (Simeone et al. 1997). Cabe también indicar el efecto de los arácnidos de familias Thomisidae y Salticidae en las poblaciones de R. buoliana; depredadores activos encontrados incluso dentro de los brotes o utilizando el pupario de $R$. buoliana para incubar sus huevos. Familias como Theridiidae, Anyphaenidae y Clubionidae esperan sus presas en las redes. Además de estas familias, cabe destacar el efecto de insectos coleópteros como Coccinellidae y Carabidae (Lanfranco et al. 1998). Sin embargo, aparte de la estimación de C. barbata, se desconoce el efecto real de los depredadores, ya que además de no tener un registro numérico, suelen ser muy generalistas (Simeone et al. 1997).

\section{ESTADO ACTUAL DEL CONTROL BIOLÓGICO DE} LA POLILLA DEL BROTE DEL PINO

La presencia de dos o más especies de parasitoides en el control de una especie plaga, así como la presencia de hiperparasitoides, depredadores e incluso especies herbívoras competidoras con la especie plaga actúan aumentando la complejidad de la comunidad y por lo tanto tiene un efecto en el equilibrio del sistema (Morin 1999, Montoya et al. 2006). Es importante conocer los procesos que alteran la dinámica de cada nivel trófico, ya que del resultado de los mismos depende la supresión de la especie plaga (Höller et al. 1993, Müller et al. 1999, van Veen 2006).

La aparición del hiperparasitoide $P$. tristis en el sistema de control de la polilla del brote en el año 2005 trajo consigo muchas interrogantes, una de ellas consistía en conocer cuál era el estado de control de $R$. buoliana en la zona de mayor impacto de la plaga, la región del Biobío. A partir del año 2006, en el laboratorio de entomología de la Universidad Austral de Chile, con ayuda de CONAFChillán (Laboratorio de Entomología y Centro de Semillas, Genética e Investigaciones Entomológicas), se realizó una investigación cuyos resultados iban encaminados al esclarecimiento de la situación del control de la polilla del brote. Utilizando las tablas de vida de Aguilar (2001) y datos obtenidos por Ide y Lanfranco (1994), CONAF (2006) y CPF (2010), se obtuvieron las curvas de supervivencia y mortalidad mostradas en la figura 2.

Cuando $R$. buoliana está bajo el efecto de los parasitoides su curva de supervivencia es de tipo III, es decir, los mayores índices de supervivencia se producen en los primeros estados y la mayor mortalidad en los últimos (figura 2). La curva de mortalidad, sin el efecto de los parasitoides, muestra una mayor supervivencia en los estados finales y una mayor mortalidad en los primeros estados, hecho comprobado por estudios anteriores donde el índice de mortalidad era muy alto en los primeros estados, de hasta un 50 \% (Ide y Lanfranco 1994, Gotelli 1998). Además, donde confluyen las curvas coincide con el estadío larvario más susceptible a la parasitación, asî como al efecto de la resina que produce la planta ante el

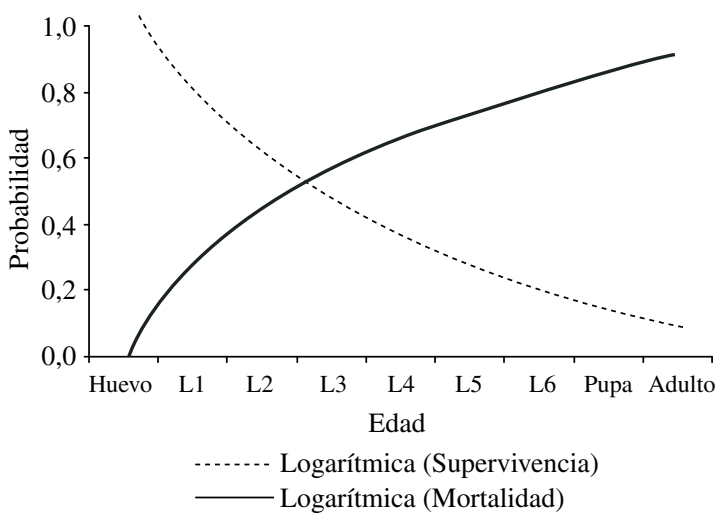

Figura 2. Curvas de supervivencia y mortalidad (escala logarítmica) de Rhyacionia buoliana (modelos a partir de Ide y Lanfranco 1994, Aguilar 2001, CONAF 2006 y CPF 2010).

Survival and mortality curves (logarithmic scale) of Rhyacionia buoliana (models from Ide and Lanfranco 1994, Aguilar 2001, CONAF 2006, CPF 2010). 
ataque de las larvas L2. Estos resultados coinciden con lo esperado, ya que el estadío L2 de R. buoliana corresponde a las larvas que han sobrevivido a la mortalidad natural (figura 2) (Ide y Lanfranco 1994).

Desde la aparición de P. tristis se han estado realizando en colaboración con el Instituto Nacional de Investigaciones Agropecuarias (INIA) trabajos para identificar su rol en el complejo parasitoide principal y cómo puede afectar el desempeño de $O$. obscurator. Para ello se han realizado crianzas y experimentos de material de predios de la región del Biobío, fundamentalmente en el marco de un proyecto fondo SAG (C4-92-14-19) (INIA 2007). Con los primeros datos se obtuvo información valiosa del comportamiento de $P$. tristis como hiperparasitoide, ya que se obtuvieron adultos de $P$. tristis desde pupas de $O$. obscurator y de T. platensis. Al analizar los registros por separado se observa una coordinación entre el ciclo de $O$. obscurator y el de $P$. tristis, emergiendo los adultos de este último justo al final del ciclo de $O$. obscurator, cuando ya han emergido la mayoría de los individuos adultos de T. platensis (figura 3) (CPF 2010).

Es probable que, por su carácter oportunista, T. platensis haya adaptado su ciclo de tal manera que, al emerger los adultos antes, éstos evitan el ataque de P. tristis. La emergencia de machos y hembras se produce entre $10 \mathrm{y}$

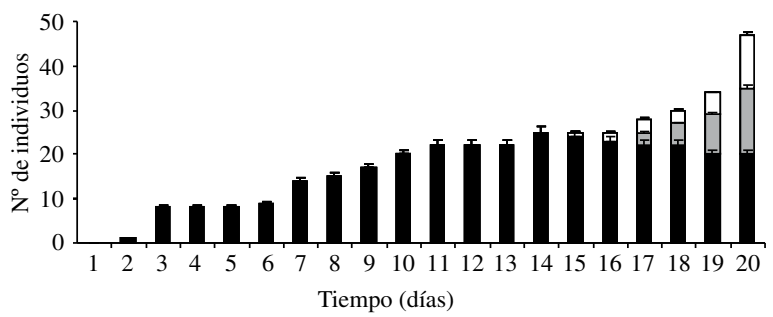

$\square$ Total $\mathrm{N}^{\circ}$ adultos eclosionados Orgilus obscurator $(\overline{\mathrm{x}} \pm \mathrm{SD})$

$\square \mathrm{N}^{\circ}$ de Perilampus tristis adultos eclosionados de pupas de Orgilus obscurator $(\overline{\mathrm{x}} \pm \mathrm{SD})$.

$\mathrm{N}^{\circ}$ de pupas de Orgilus obscurator presentes $(\overline{\mathrm{x}} \pm \mathrm{SD})$

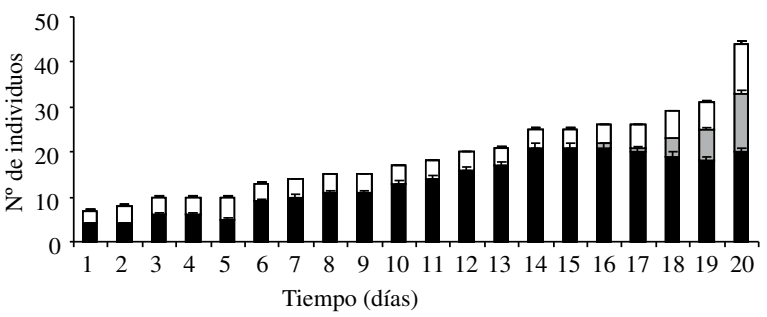

Total $\mathrm{N}^{\mathrm{o}}$ adultos eclosionados Temelucha platensis $(\overline{\mathrm{x}} \pm \mathrm{SD})$.

$\square \mathrm{N}^{\circ}$ de Perilampus tristis adultos eclosionados de pupas

de Temelucha platensis $(\overline{\mathrm{x}} \pm \mathrm{SD})$.

$\mathrm{N}^{\circ}$ de pupas de Temelucha platensis presentes $(\overline{\mathrm{x}} \pm \mathrm{SD})$.

Figura 3. Número de pupas y adultos de Perilampus tristis, Orgilus obscurator y Temelucha platensis criados en laboratorio (Fuente: CPF 2010).

Number of pupas and adults of $P$. tristis, $O$. obscurator and T. platensis reared under laboratory conditions (source: CPF 2010).
15 días antes que $O$. obscurator, viéndose menos afectados en su población por el efecto de $P$. tristis (figura 3) (CPF 2010).

La información de emergencia de adultos de los tres parasitoides provenientes de crianzas de larvas de la región del Biobío (CONAF 2006, CPF 2010), en una serie temporal de diez años, muestra una tendencia a la estabilidad en las poblaciones de R. buoliana y P. tristis (figura 4). No sucede lo mismo con $O$. obscurator y $T$. platensis, aunque estos datos deben interpretarse con cautela, ya que no se conoce en detalle el tipo de interacciones que pueda darse entre estos dos parasitoides y la especie plaga, la influencia del tamaño del rodal y de otros rodales circundantes, ni si esta tendencia vaya a continuar en el tiempo.

\section{ACCIONES FUTURAS}

El futuro de este complejo de parasitoides y del control biológico de $R$. buoliana actualmente pasa por descubrir las interacciones existentes entre las tres especies de parasitoides que presentan mayor importancia, no sólo por los niveles de parasitismo logrados por las tres, sino porque son las que actúan en el estadío más susceptible de $R$. buoliana y además una de ellas, $O$. obscurator, es la seleccionada desde hace veinte años para llevar a las poblaciones de $R$. buoliana bajo niveles de daño económico (Lanfranco et al. 1991, 1994).

El masivo uso de $O$. obscurator extraído desde lugares en donde fueron previamente liberados podría estar actuando en detrimento de la eficacia del parasitoide y sus poblaciones. Una de las líneas de investigación sugeridas en este sentido es el estudio genético poblacional del parasitoide para descartar posibles procesos de inbreeding, cuellos

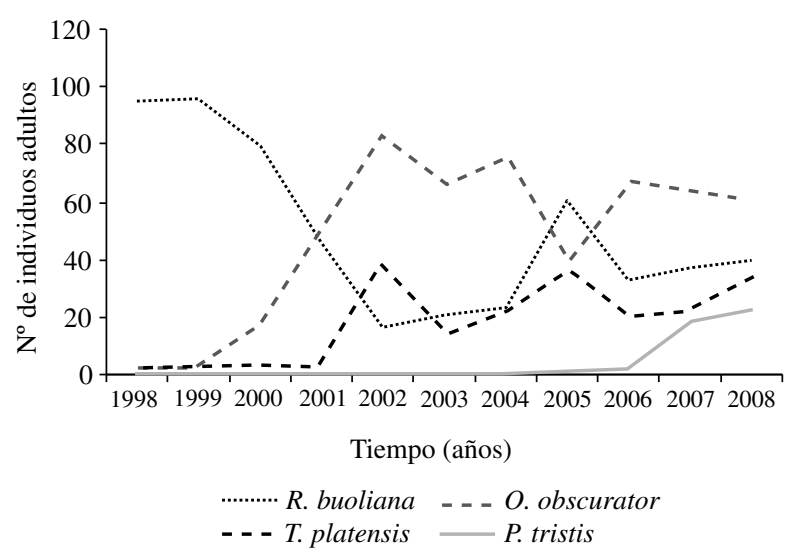

Figura 4. Dinámica poblacional del complejo de parasitoides asociado a Rhyacionia buoliana para el periodo 1998-2008 (Fuente: CONAF 2006, CPF 2010).

Population dynamics of the parasitoid complex associated to Rhyacionia buoliana for the period 1998-2008 (source: CONAF 2006, CPF 2010). 
de botella u otros, que pudieran afectar la efectividad de O. obscurator (Paredes et al. 2007).

Es probable que tanto $T$. platensis como $P$. tristis estén actuando de manera oportunista y afectando el control de O. obscurator en algunas zonas del área de liberación. En todo caso, debería hacerse una prospección en toda la zona de distribución de $R$. buoliana para estimar las densidades poblacionales actuales por región y así tratar de responder a las interrogantes que se han planteado en estos últimos años sobre el control de esta plaga.

Con los datos obtenidos hasta el momento se ha avanzado en el conocimiento del comportamiento del nivel trófico superior, es decir $P$. tristis, respecto a sus principales presas u hospederos, los parasitoides primarios $O$. obscurator y T. platensis, ya que si bien no parece tener una clara preferencia por uno de los dos parasitoides, sólo se ha encontrado este hiperparasitoide en larvas previamente parasitadas, lo que descartaría un comportamiento facultativo.

Uno de los resultados que llama la atención es la estrecha coordinación que existe entre el ciclo de $O$. obscurator y P. tristis, a pesar de que este último ataca por igual a ambos parasitoides primarios. Una posible explicación es que T. platensis haya adecuado su ciclo ante los cambios que se producen por la acción del hiperparasitoide, siendo su comportamiento más plástico que el de $O$. obscurator.

Una de las propuestas formuladas en los últimos años consiste en ayudar a $O$. obscurator con la implementación de otros parasitoides que actúen en estadíos en los que no interfiera con él, como el parasitoide de pupas Tetrastichus turionum Htg. (Hym.: Eulophidae) (Huerta et al. 2004). Sin embargo, debe tenerse en cuenta la posible aparición de competencia entre $T$. turionum y $P$. fuscipes, ya que este último, por ser generalista, es más efectivo en el corto plazo (Lanfranco y Cerda 1987, Stiling y Cornelissen 2005).

Abrir de nuevo el debate de elección entre liberaciones múltiples o simples en control biológico no es actualmente una opción. Es posible que la liberación de nuevos parasitoides genere nuevas tramas e interacciones cuya finalidad no sea otra que disminuir el control de $R$. buoliana (Stiling y Cornelissen 2005).

Para poder responder a esta hipótesis y conocer el posible efecto que cada uno de los comportamientos de los parasitoides tiene en el sistema multiespecífico, se propone la utilización de modelos teóricos de poblaciones. A su vez, estos modelos deben incorporar factores múltiples, ya sean intrínsecos del complejo como extrínsecos de localidad, clima, metodología de muestreo, edad de las plantaciones, etc. La finalidad de estos modelos no es otra que poder predecir cambios en las poblaciones de la especie plaga en función de los resultados obtenidos, pudiendo así estimar si el control biológico es efectivo y cuáles podrían ser los factores que debilitarían dicho control.

Otra manera de detectar las interacciones entre las especies es realizar ensayos de laboratorio que demuestren claramente la condición de hiperparasitoide facultativo u obligado de $P$. tristis, ofertándole a los adultos larvas de
$R$. buoliana parasitadas por $O$. obscurator, parasitadas por $T$. platensis y parasitadas por $O$. obscurator y $T$. platensis. Asimismo, para T. platensis, larvas no parasitadas, parasitadas con $O$. obscurator solamente y parasitadas con $O$. obscurator y $P$. tristis. De esta forma se podría conocer si estos parasitoides compiten entre sí, interfieren con el control de O. obscurator o si todas contribuyen al control de $R$. buoliana.

Otro punto a considerar es el efecto que tienen los cambios climáticos y ambientales en el control biológico. Se sabe que las especies de insectos plaga son capaces de responder rápidamente ante cambios medioambientales, sin embargo, el efecto que esta respuesta poblacional tiene sobre las poblaciones de sus biocontroladores es todavía desconocido. Por ello, éste es un factor a tener en cuenta al pensar en la efectividad del control biológico en el largo plazo (Stireman et al. 2005, Hance et al. 2007).

\section{AGRADECIMIENTOS}

Este trabajo se realizó bajo el auspicio de la beca de doctorado MAEC- AECI (periodo 2006-2007) junto al apoyo del Laboratorio de Entomología, Centro de Semillas, Genética e Investigaciones Entomológicas (CONAF: Chillán), el Fondo SAG (C4-92-14-19) y la Dirección de Investigación y Desarrollo de la Universidad Austral de Chile. Agradecemos el apoyo de los integrantes del Laboratorio de Entomología del Instituto de Silvicultura de la Universidad Austral de Chile.

\section{REFERENCIAS}

Aguilar AM. 2001. Tabla de vida y factores de mortalidad para una generación de Rhyacionia buoliana (Schiff) en una localidad de la X Región. Chile. Tesis de Magíster. Valdivia, Chile. Facultad de Ciencias, Universidad Austral de Chile. 86 p.

Arthur AP, AJ Juliet. 1961. The introduced parasites of the European Pine Shoot Moth, Rhyacionia buoliana (Schiff.) (Lepidoptera: Olethreutidae), with a critical of their usefulness as control agents. Can. Entomol. 93(4): 297-312.

Arthur AP, JER Stainer, AL Turnbull. 1964. The interactions between Orgilus obscurator (Ness) (Hymenoptera: Braconidae) and Temelucha interruptor (Hymenoptera: Ichneumonidae), parasites of the Pine Shoot Moth, Rhyacionia buoliana (Schiff.) (Lepidoptera: Olethreutidae). Can. Entomol. 96: 1030-1034.

BIOforest. 1995. Efecto de Trichogramma embryophagum, T. exiguum y $T$. pretiosum sobre el ataque producido por la población de Rhyacionia buoliana Denis y Schiffermüller mediante liberaciones en Terreno. Valdivia. Chile. División de Protección Fitosanitaria. 13 p. (Informe Técnico BFP $\mathrm{N}^{\mathrm{o}}$ 010).

Boettner GH, JS Elkinton, CJ Boettner. 2000. Effects of a biological control. Introduction on three nontarget native species of Saturniid moths. Conserv. Biol. 14: 1798-1806. 
Botto E. 1998. Polilla europea del Brote del Pino en la Patagonia. Presencia, impacto económico y posibilidades de Control Biológico. Idia 21: 129-131.

Brèthes J. 1917. Descripción de dos nuevos himenópteros de Buenos Aires. Physis 3: 90-91.

Buitenhuis R, LEM Vet, G Boivin, J Brodeur. 2005. Foraging behavior at the fourth trophic level: A comparative study of host location in aphid hyperparasitoids. Entomol. Exp. Appl. 114: 107-117.

Cerda LA, C Jana-Saenz, M A Beèche. 1985. Detección de la "Polilla del Brote" en Chile: Rhyacionia buoliana (Schiffermüller, 1776) (Lepidoptera: Tortricidae). Bol. Soc. Biol. Concepción. 56: 161-162.

Cerda LA, C Jana-Saenz, O Puentes. 1986. Ciclo de vida en Chile de Rhyacionia buoliana (Schiff.) (Lepidoptera, Tortricidae). Bol. Soc. Biol. Concepción. 57: 201-203.

Cerda LA, MA Aguilar, MA Beèche. 1988. Insecticidas para el control de Rhyacionia buoliana (Schiff.) (Lepidoptera: Tortricidae): Ensayo de laboratorio. Bosque 9(1): 61-63.

Clausen CP. 1940. The immature stages of the Eucharitidae (Hymenoptera). J Wash Acad Sci. 30: 504-516.

CONAF (Corporación Nacional Forestal, CL). 2006. Informe final: Control biológico de la polilla del brote en pequeñosmedianos predios de la VIII Región. Santiago, Chile. CONAF. $122 \mathrm{p}$.

CPF (Controladora de Plagas Forestales S. A., CL). 2010. Estudio de la biología e impacto potencial de Perilampus tristis sobre la interacción entre Rhyacionia buoliana y Orgilus obscurator. CPF. Santiago, Chile. 91 p.

Cruttwell R. 1998. Biological control of weeds. Annu. Rev. Entomol. 43: 369-393.

Darling DC. 1996. Generic concepts in the Perilampidae (Hymenoptera: Chalcidoidea): An assessment of recently proposed genera. J. Hym. Res. 5: 100-130.

Darling DC, H Roberts. 1999. Life history and larval morphology of Monacon (Hym: Perilampidae) parasitoids of ambrosia beetles (Coleptera: Platypodidae). Can. J. Zool. 77: $1768-1782$.

Day WH. 2005. Changes in abundance of native and introduced parasites (Hymenoptera: Braconidae), and of the target and non-target plant bug species (Hemiptera: Miridae), during two classical biological control programs in alfalfa. Biol. Control. 33: 368-374.

Donoso C, A Lara. 1996. Utilización de los Bosques Nativos en Chile: Pasado, Presente y Futuro. In Armesto JJ, C Villagrán, MK Arroyo eds. Ecología de los bosques nativos de Chile. Santiago, Chile. Editorial Universitaria. p. 363-404.

Gauld ID, B Bolton. 1988. The Hymenoptera. Oxford, England. Oxford University Press. 332 p.

Gauld ID, SR Shaw. 1995. The Ichneumonoid Families. In Hanson PE, ID Gauld eds. The Hymenoptera of Costa Rica. Oxford. Oxford Science Publications. The Natural History Museum. London, England. Oxford University Press. p. 389-463.

Gayoso J. 1996. Costos ambientales en plantaciones de Pinus radiata D. Don. Bosque 17(2): 15-26.

Gerding MP, CP Torres. 2001. Parasitoide de huevos de polillas Trichogramma: Insecto benéfico para el control de plagas. Boletín Informativo. Instituto de Investigaciones Agropecuarias Centro Regional de Investigación Quilamapu. Consultado 9 sep. 2008. Disponible en: http//www.controlbiologicochile. $\mathrm{cl} /$ content/blogsection/15/137/
Gotelli NJ. 1998. A primer of Ecology. Sunderland, Massachusetts, USA. Sinauer Associates. 236 p.

Hance T, J van Baaren, P Vernon, G Boivin. 2007. Impact of Extreme Temperatures on Parasitoids in a Climate Change Perspective. Annu. Rev. Entomol. 52: 107-126.

Hanson PW, J LaSalle. 1995. The Chalcidoid families. In Hanson PE, ID Gauld eds. The Hymenoptera of Costa Rica. Oxford. Oxford Science Publications. The Natural History Museum. London, England. Oxford University Press. p. 266-388.

Hawkins BA, MB Thomas, ME Hochberg. 1993. Refuge theory and biological control. Science 262: 1429-1431.

Heeley T, RI Alfaro, L Humble. 2003. Distribution and life cycle of Rhyacionia buoliana (Lepidoptera: Tortricidae) in the interior of British Columbia. Jour. Entomol. Soc. Brit. Columbia 100: 19-25.

Heraty JM, DC Darling. 1984. Comparative morphology of the planidial larvae of Eucharitidae and Perilampidae (Hymenoptera: Chalcidoidea). Syst Entomol. 9: 309-328.

Hoffman JH, FAC Impson, VC Moran, D Donnelly. 2002. Biological control of invasive golden wattle trees (Acacia pycnantha) by a gall wasp, Trichilogaster sp. (Hymenoptera: Pteromalidae), in South Africa. Biol. Control. 25: 64-73.

Hoelmer KA, AA Kirk. 2005. Selecting arthropod biological control agents against arthropod pests: Can the science be improved to decrease the risk of releasing ineffective agents? Biol. Control. 34: 255-264.

Höller C, Borgemeister C, Haardt H, and Powell W. 1993. The relationship between primary parasitoids and hyperparasitoids of cereal aphids: an analysis of field data. J. Anim. Ecol. 62: 12-21.

Hougardy E, JC Grégoire. 2003. Cleptoparasitism increases the host finding ability of a polyphagous parasitoid species Rhopalicus tutela (Hymenoptera: Pteromalidae). Behav. Ecol. Sociobiol. 55: 184-189.

Howarth FG. 1991. Environmental impacts of classical biological control. Annu. Rev. Entomol. 36: 485-509.

Huerta A, C Pérez. 1997. Ciclo de vida de la Polilla del Brote del Pino (Rhyacionia buoliana Den. et Schiff.) en la VII Región de Chile. Bol. San. Veg. Plagas. 23(3): 385-391.

Huerta A, F Robredo, J Diez, JA Pajares. 2004. Life-cycle characteristics of Tetrastichus turionum a parasitoid of the European pine shoot moth, Rhyacionia buoliana. JEN 128: 628-632.

Hunter MS, TR Collier, SE Kelly. 2002. Does an autoparasitoid disrupt host suppression provided by a primary parasitoid? Ecology 83: 1459-1469.

Ide S, D Lanfranco. 1994. Fluctuaciones poblacionales de Rhyacionia buoliana (Lep: Tortricidae) en una plantación de Pinus radiata en la X Región. Bosque 15(2): 39-44.

Ide S, D Lanfranco. 2001. Longevidad de Orgilus obscurator Ness. (Hymenoptera: Braconidae) en presencia de diferentes fuentes de alimento. Rev. Chil. Hist. Nat. 74: 469-475.

Ide S, D Lanfranco, C Gouet. 2007. Detección de superparasitismo y multiparasitismo sobre larvas de Rhyacionia buoliana (Lepidoptera Tortricidae) en las Regiones Octava y Novena de Chile. Bosque 28(1): 57-64.

INIA (Instituto Nacional de Investigaciones Agropecuarias, CL). 2007. Biología e impacto de Perilampus en interacción Rhyacionia-Orgilus. Consultado 23 may. 2009. Disponible en http://www.controlbiologicochile.cl/content/view/35/130/

INIA/SAG (Instituto Nacional de Investigaciones Agropecuarias, CL/Servicio Agrícola y Ganadero, CL). 1993. Control 
biológico de Rhyacionia buoliana Dennis \& Schiffermüller. Valdivia Chile. Instituto de Investigaciones Agropecuarias, Estación Experimental Remehue. 87 p. (Convenio INIA/ SAG Ministerio de Agricultura, Informe Final).

Jaloux B, C Errard, N Mondy, F Vannier, J-P Monge. 2005. Sources of chemical signals which enhance multiparasitism preference by a cleptoparasitoid. J. Chem. Ecol. 31(6): 1325-1337.

Jaloux B, JP Monge. 2006. Kairomone stimulates increased probes and host stings in a cleptoparasitoid. Physiol. Entomol. 31: 197-200.

Jaloux B, M Deshayes, JP Monge. 2007. Learning affects the preference for parasitized host of the facultative kleptoparasitoid Eupelmus vuilleti. Behav. Ecol. Sociobiol. 31: 357-364.

Juillet JA. 1960. Immature stages, life histories, and behavior of two hymenopterous parasites of the European pine shoot moth Rhyacionia buoliana (Schiff.) (Lepidoptera: Olethreutidae). Can. Entomol. 92: 342-246.

Kehrli P, M Lehmann, S Bacher. 2005. Mass-emergence devices: a biocontrol technique for conservation and augmentation of parasitoids. Biol. Control. 32: 191-199.

Lanfranco D, M Cerda. 1987. Coccygommimus fuscipes (Hym: Ichneumonidae): Un parasitoide nativo de la Polilla del Brote Rhyacionia buoliana (Lep: Tortricidae). Bosque 7 (1): 36-37.

Lanfranco D, JF Büchner, MA Aguilar, RA Horcos. 1991. Parasitoides nativos en el control de la polilla del brote del pino (Rhyacionia buoliana): avances en la identificación del complejo y de sus estrategias de desarrollo. Bosque 12(1): 69-74.

Lanfranco D, MA Aguilar, RA Horcos, JF Büchner. 1994. Parasitoides nativos en el control de la polilla del brote del pino (Rhyacionia buoliana): ¿Un complejo funcional? Bosque 15(1): 15-26.

Lanfranco D, S Ide, E Rojas, C Ruiz, R Carrillo, C Martínez, P Jofré, A Simeone, R Schlater, JC Valencia, R Calderón. 1998. Biological control agents in management of pine shoot moth: Studies, findings and expectations. Biocontrol News and Information 19(1): 33-34.

Linch LD, AR Ives, JK Waage, ME Hochberg, MB Thomas. 2002. The risks of biocontrol: Transient impacts and minimum nontarget densities. Ecological Appl. 12(6): 1872-1882.

Louda SM, D Kendall, J Connor, D Simberloff. 1997. Ecological effects of an insect introduced for the biological control of weeds. Science 277: 1088- 1090.

McFadyen REC. 1998. Biological control of weeds. Annu. Rev. Entomol. 43: 369-393.

Mills N. 2005. Selecting effective parasitoids for biological control introductions: Codling moth as a case study. Biol. Control. 34: 255- 264.

Montoya, JM, Pimm, SL, and Solé SV. 2006. Ecological networks and their fragility. Nature. 442: 259-264.

Morin, PJ. 1999. Community Ecology. Blackwell Science. Massachusetts. USA. 424 p.

Müller CB, ICT Adriaanse, R Belshaw, HCJ Godfray. 1999. The structure of an aphid-parasitoid community. J. Anim. Ecol. 68: 346-370.

Paredes M, V Becerra, E Cisternas, M Gerding. 2007. Detección de polimorfismo en poblaciones de Orgilus obscurator (Nees) presentes en Chile. AgroCiencia 23(2): 65-72.

Parker HL. 1924. Recherches sur les formes post-embryonaires des Chalcidiens. (In French.). Ann. Soc. Entomol. France 93: 261-379.

Parker JD, DE Burkepile, ME Hay. 2006. Opposing effects of native and exotic herbivores on plant invasions. Science 311: 1459-1461.
Pintureau B, M Gerding, E Cisternas. 1999. Descriptions of three new species of Trichogrammatidae (Hymenoptera) from Chile. Can. Entomol. 131: 53-62.

Robredo FJ. 1978. Contribución al conocimiento de la bioecología de Rhyacionia buoliana DEN. y SCHIFF., 1776 (Lep., Tortricidae). II. Estudio de los estados inmaduros: puesta, oruga y crisálida. Bol. Serv. Plagas. 4: 69-88.

Schooler SS, AR Ives, J Harmon. 1996. Hyperparasitoid aggregation in response to variation in Aphidius ervi host density at three spatial scales. Ecol. Entomol. 21: 249-258.

Schröder D. 1974. A study of the interactions between the internal larval parasites of Rhyacionia buoliana (Lepidoptera: Olethreutidae). Entomophaga 19(2): 145-171.

Simberloff D, P Stiling. 1996. Risks of Species Introduced for Biological Control. Biol. Conserv. 78: 185-192.

Simeone A, JC Valencia, R Schlatter, D Lanfranco, S Ide. 1997. Depredación de aves sobre larvas de Rhyacionia buoliana (Schiff.) (Lepidoptera: Tortricidae) en plantaciones jóvenes de Pinus radiata D. Don en el sur de Chile. Bosque 18(2): 67-75.

Smith HH. 1912. The chalcidoid genus Perilampus and its relations to the problem of parasite introduction. United States Department of Agriculture. Bureau of Entomology. 69 p.

Smith SM. 1996. Biological Control with Trichogramma: Advances, Successes, and Potential of Their Use. Annu. Rev. Entomol. 41: 375-406.

Stiling P, T Cornelissen. 2005. What makes a successfull biocontrol agent? A meta-analysis of biological control agent performance. Biol. Control. 34: 236-246.

Stireman JO, LA Dyer, DH Janzen, MS Singer, JT Lill, RJ Marquis, RE Ricklefs, GL Gentry, W Hallwatch, PD Coley, JA Barone, HF Greeney, H Connahs, P Barbosa, HC Morais, IR Diniz. 2005. Climatic unpredictability and parasitism of caterpillars: Implications of global warming. PNAS 48: 17384-17387.

Sullivan DJ, W Völkl. 1999. Hyperparasitism: Multitrophic ecology and behavior. Annu. Rev. Entomol. 44: 291-315.

Tanton MT, JSO Epila. 1984. Description of the planidium of Perilampus tasmanicus Cameron (Hymenoptera: Chalcidoidea), a hyperparasitoid of larvae of Paropsis atomaria Olivier (Coleoptera: Chrysomelidae) J. Aust. Ent. Soc. 23: 149-152.

Taylor AD. 1988. Parasitoid competition and the dynamics of host-parasitoid models. Am. Nat. 132: 417-436.

Tezze AA, EN Botto. 2004. Effect of cold storage on the quality of Trichogramma nerudai (Hymenoptera: Trichogrammatidae). Biol. Control. 30: 11-16.

Torres CP, MP Gerding. 2005. Uso de Trichogramma como controlador biológico. Boletín Plagas y Enfermedades. Consultado 9 sept. 2008. Disponible en: http//www.controlbiologicochile.cl/content/blogsection/15/137/

Townes H. 1971. The genera of Ichneumonidae. Part 4: Cremastinae to Diplazontinae. Mem. Amer. Ent. Inst. 17: 23-24.

van Driesche RG, TSJr Bellows. 1996. Biological Control. New York, USA. Chapman and Hall. 539 p.

van Lenteren JC, J Bale, F Bigler, HMT Hokkanen, AJM Loomans. 2006. Assessing risks of releasing exotic biological control agents of arthropod pests. Annu. Rev. Entomol. 51: 609-634.

van Veen FJF, Morris RJ, HCJ Godfray. 2006. Apparent competition, quantitative food webs, and the structure of phytophagous insect communities. Annu. Rev. Entomol. 51: 187-208.

Wahl D, Gauld I. 1998. The cladistics and higher classification of the Pimpliformes (Hymenoptera- Ichneumonidae). Syst. Entomology 23: 265-298.

Recibido: 11.01 .10

Aceptado: 03.06.10 